\title{
The Light Quantity Hypothesis*
}

\author{
New Ideas as to the Atomic Nature of Radiant Energy Which Promises Solutions of Many Difficulties
}

\section{By Prof. D. F. Comstock, of the Massachusetts Institute of Technology}

Within the last few years the idea has been gaining importance that the light radiated from a luminous body is not emitted continuously, but in small indivisible "atoms of energy." This notion is totally unexpected rom the standpoint of a decade ago, but evidence of one form or another is piling up so fast that it appears at present highly probable this view will, in the end, prove to represent the truth.

The idea was first put forward by a physicist of international fame, Prof. Max Planck, of the University of Berlin. He was led to this conclusion through his study of the so-called laws of radiation.

$$
\text { RADIATION FROM A BLACK BODY. }
$$

In order to make a discussion of this hypothesis at all clear, it is necessary to speak of the phenonema of radiation in general. The simplest type of radiation which we know is that which a so-called "black body" gives off when heated. Perhaps the simplest black body we could take would be a tin cup covered with lamp black on the outside and filled with hot water. This body gives off heat rays in all directions which, as far as we know, differ in no way whatever from light rays except that they are on the average of a lower frequency, i. e., they bear the same qualitative relation to light rays that the lowest note in the pian scale bears to the highest. These rays do not affect the eye because the retina is not so constructed that it can detect such relatively slow vibrations. The laws of propagation of the waves and their general characteristics are apparently, however, exactly the same as those of light rays.

Now, if we suppose the cup filled with something besides water and to be slowly heated hotter and hotter, wo should notice two significant changes in this radiation which spreads out in all directions from the cup. In the first place, as it gets hotter, more energy will be radiated in a unit of time, and second, such energy as is radiated will have a higher average frequency than before. Making a sound analogy, we might say that this corresponds to the sounds given off being louder and of higher pitch. If this heating proces goes on indefinitely the frequency will soon be high enough so that the retina of the eye will be affected by the rays and the object will be "red hot."

$$
\text { WHERE WIEN'S ForMULA FaILS. }
$$

Now the exact way in which the radiation of a black body changes with increasing temperature, has been studied very carefully and several theoretical formulm have been found which agree more or less closely with have been found which agree more or less closely with
experimental results. Perhaps the most successful of these is the so-called formula of Wien which was derived from the fundamental conceptions of atomic theory. It is almost certainly true that the radiation from a hot body is caused by the chaotic to-and-fro motion of the atoms or molecules of which the substance is composed, and the general distribution of motion among the vibrating molecules or atoms is pretty well among the vibrating molecules or atoms is pretty well
known at the present time. Wien obtained his formu'a known at the present time. Wien obtained his formu'a
by supposing that each molecule radiated energy in a way which depended only on its velocity at the instan in question.

Now, although Wien's formula certainly contains some element of truth, it fails conspicuously at high temperatures. This failure has been realized for number of years, but no suggestion was forthcoming as to why it failed.

This failure of Wien's formula is by no means an isolated fact of small importance. The ablest thinkers in theoretical physics all over the world have con sidered at great length the theory of radiation, and although there has been more or less complete agreoment among them, their results are not accurately in agreement with the results of experiment. Indeed. decade ago it was generally admitted that somethin was wrong with the fundamental notions the theory. PLANCK's "ATOMS OF ENERGY."

In about 1905, however, Prof. Planck came forward with an entirely new hypothesis. By one of the mys terious mutations which we call "inspirations of genius," he came to the conclusion that if we were to get a theory consistent with experiment, we must assume that the atoms do not radiate energy continuously, but radiate only in small discrete quantities-"atoms of energy" on might say. From this point of view the oscillating part of an atom cannot possess any amount of energy, but only an amount which is a definite multiple of a fundamental unit of energy.

* Reproduced from science Conspectus.
This is an extraordinary assumption and is in a sense sudden jump in physical theory, but Planck showed that when one developed this hypothesis by well-know and rigid methods, an equation was obtained which differed slightly from Wien's formula and which wa much more hopeful in its form. As a matter of fact, careful experiments have shown that as accurately as measurements have been carrict

exact for all temperature

It is an interesting and suggestive fact that Wien' formula appears as a special case of Planck's whe the temperature is not too high. In other word Planck's formula contains apparently all the trut that is in Wien's and enough more to make it an exact representation of the fact. It is also an interesting side-light on the rapidity with which apparently remote scientific hypotheses may react on the industrial world, that Planck's formula is already beginning to have an important effect on interpreting the results of the so-called optical pyrometers so extensively used in measuring the high temperatures of furnaces.

So extraordinary is the assumption of discrete quantities of energy that, were it only applicable to the theory of radiation, the conception would not be as impressive as it has grown to be within the last few years, but curiously enough there is totally independen experimental evidence for it. Other results seem to indicate that light and similar wave motions propagat in the form of units of energy.

$$
\text { THE SHOWER-OF-STONES ANALOGY. }
$$

It is a well-known fact in physies that if a current is passed through any vacuum tube in which the vacuum is very high, a stream of electrons proceeds from the negative terminal in the tube and travels in straight lines. This stream of electrons constitutes what w know as the cathode rays. When these rays strike an obstacle, X-rays are emitted from the point struck just as sound would be emitted from a barn door struck by a shower of stones. These X-rays are considere by a shower of stones. These X-rays are considered
to be of the wave-motion type of disturbance and not a stream of particles. The shower-of-stones-sound analogy -is, therefore, quite accurate since sound is a wave motion. The analogy, however, fails of further application for it is found that when these $\mathrm{X}$-rays strike a metal they give rise to a stream of cathode rays (i. e., a stream of electrons) which are called the "secondary cathode rays," and these cathode rays give rise to other X-rays. If the stone analogy were to be complete, the sound from the point struck by the stone would have to cause a stream of stones to come out of any substance which the sound struck.

Now, the curious fact noticed about these electrons which spring out of a metal when it is struck by X-rays is that their maximum speed does not seem to depend on how intense the $\mathrm{X}$-rays are, nor does it depend on the nature of the metal struck by them. If a piece of metal is taken farther away from the source of rays, so that the energy of the rays falling on it is less, it is found that fewer electrons come out of the metal in a unit time, but those that do come out have the same maximum velocity that they had when the metal was nearer.

Now, a little thought will show that this result is totally inexplainable on the older ideas of radiant energy, for the energy of the outcoming electrons is evidently derived from the energy of the striking X-rays, and as this energy spreads out indefinitely and gets weaker, it is to be expected that all effects produced by the enorgy would slowly dwindle away. This is not true, howover; the emitted electrons come out with the samo maximum speed as when far more energy struck them

Now, on the "light quantity hypothesis," the results are quite clear, for if the energy of the X-rays is really in the form of little "particles" of energy, then at greater distances from the source of the rays, the only greater distances from the source of the rays, the only
difference is that the particles would be farther apart on the average. They are spread out more, but each has the same amount of energy and each is, therefore, capable of giving an impulse of the same strength to the electron in the metal which it happens to strike as it would have given had the metal been nearer; so that the experimental results, totally inexplainable on the older idea of continuous energy radiation, become readily explained when this "light quantity hypothesis" is applied.

$$
\text { "ABSOLUTE ZERo" AND TIE NEW HYPOTHESIS. }
$$

Besides these two rather striking examples of the evidence for the light quantity hypothesis there are a number of others in which it is either definitely suggested by experiments or dimly foreshadowed. One set of results which seems to promise evidence in favor of the hypothesis is obtained in modern low temperature research. As is well known, the theoretical limit in diminishing temperature is the so-called "absolute zero." This is the temperature at which all atomic energy, or molecular energy of vibration would cease and is located at approximately $273 \mathrm{deg}$. Cent. below zero or $460 \mathrm{deg}$. Fahr. below zero. It is only very recently that temperatures at all approximating the absolute zero have been obtained but now we have results on the electrical resistance of platinum wire measured at $1.5 \mathrm{deg}$. Cent. above the absolute zero. At this temperature air and even hydrogen are solid. Now, at these very low temperatures there are certain unexpected anomalies in both electrical and heat phenomena.

The so-called specific heat of substances, which from the older ideas should approach a certain definite constant as the temperature approaches the absolute zero, changes in quite an unexpected way as the temperature diminishes, for at these very low values it becomes so rapidly less as to approach zero instead of the contant finite value as above mentioned. It is very difficult to get any explanation of these low temperature anomalies on the older ideas of continuous energy distribution. On the energy quantity hypothesis, however, the results while not yet worked out sufficiently to be called at all certain, are, nevertheless, very suggestive and promising.

FLUORESCENCE AND THE SIZE OF THE ATOMS OF ENERGY.

Even in the domain of fluorescence an obscure question seems to be on the point of being cleared up by light quantity ideas, for it is well known in general, that fluorescent light is nearly always of lower frequency than the light which eauses the fluorescence. This is in line with the new ideas, for although not yet mentioned in this paper, the so-called atoms of energy are not all of the same size, but are larger in proportion to the frequency of light, so that the size of the "atoms" corresponding to blue light, is greater than the size corresponding to red light, and it is clear, therefore, that when a body fluoresces, under stimulation from light of any color, it cannot give off larger light atoms (i. e., bluer light) than it receives from the stimulating light, whereas it is perfectly conceivable that through some type of complex transformation, some of the ing might be left in a body and the energy quantities in the fluorescent light might be fuorescent light might be redder.

The light quantity hypothesis has not by any means been worked out so completely yet that its position and possibilities are well defined, but it seems at present full of promise, and it is being used more and more by physicists throughout the world in explaining phenomena hitherto mysterious both in the realm of radiation and in that of electricity.

Soap for Cleaning Very Dirty Hands.-It is well known that chauffeurs, mechanicians and otbers whose occupations are essentially dirty and involve soiling with axle-grease, cleanse their hands by washing them in petroleum essence or rubbing them with oil, before resorting to soap and water. This is not always practical or feasible. For this reason there have been brought out, with profuse advertisement, various special soaps, some of which are effacious, whilo others are not. It is easy to prepare such products by incorporating with the soap some kind of abrasive substance which scrape the skin and absorbs the dirty grease. Do not use sand, which imparts, on use, a very disagreeable feeling, but finely sifted sawdust or cork powder, and use a sufficient quantity, 10 per cent, for instance, taking care to rinse it thoroughly and to avoid leaving the mass to stand as long as it is liquid. Practically and on a small scale, it is best to use a fat soap. Its efficacy can be increased by adding uniformly, before using. a little grease solvent, petrol essence, benzine, denatured alcohol, but not too much, because then the mass, too fluid, deliquesces when standing. There should be undertaken, for an industrial manufacture, a study of a combination of soap, sawdust and solvent, having exactly or nearly the same density as will produce a very permanent emulsion. Without doubt, one can easily obtain this by mixing various chlorine derivatives of hydrocarbon, so as to obtain a solvent that will be non-combustible and but slightly volatile.Les Matieres Girasses, 